\title{
Étude naturalistique de la négociation des intersections et du respect des limites de vitesse chez les conducteurs âgés de 65 ans et plus
}

\section{Naturalistic study of stop-controlled intersections negotiation and respect for speed limits in drivers aged 65 and over}

\author{
Mohamed-Amine Choukou · Catherine Bluteau - Mathieu Germain Robitaille - Martin Simoneau • \\ Martin Lavallière · Thierry Moskowicz · Denis Laurendeau · Normand Teasdale
}

C IFSTTAR et Éditions NecPlus 2014

\author{
Mohamed-Amine Choukou $(\triangle)$ \\ Université Laval, Faculté de médecine, Département de kinésiologie \\ 2300, rue de de la Terrasse \\ Québec, Canada G1V 0A6 \\ e-mail : choukouamine@gmail.com \\ Catherine Bluteau $(\square)$ \\ Université Laval, Faculté de médecine, Département de kinésiologie \\ 2300, rue de de la Terrasse \\ Québec, Canada G1V 0A6 \\ e-mail : catherine.bluteau.1@ulaval.ca
}

\author{
Mathieu Germain Robitaille $(\bowtie)$ \\ Université Laval, Faculté de médecine, Département de kinésiologie \\ 2300, rue de de la Terrasse \\ Québec, Canada G1V 0A6 \\ e-mail : Mathieu.Germain-Robitaille@kin.ulaval.ca
}

Martin Simoneau $(\bowtie)$

Université Laval, Faculté de médecine, Département de kinésiologie

2300, rue de de la Terrasse

Québec, Canada G1V 0A6

e-mail : Martin.Simoneau@kin.ulaval.ca

Martin Lavallière $(\square)$

Massachusetts Institute of Technology

77 Massachusetts Avenue, Room E40-291

Cambridge, MA 02139-4307, Etats-Unis

e-mail : mlav@mit.edu

Thierry Moskowicz $(\bowtie)$

Université Laval, Département de génie électrique et de génie

informatique

Pavillon Pouliot

Québec, QC G1K 7P4

e-mail : Thierry.Moszkowicz@gel.ulaval.ca

Denis Laurendeau $(\square)$

Université Laval, Département de génie électrique et de génie

informatique

Pavillon Pouliot

Québec, QC G1K 7P4

e-mail : Denis.Laurendeau@gel.ulaval.ca

Normand Teasdale $(\bowtie)$

Université Laval, Faculté de médecine, Département de kinésiologie, PEPS

2300, rue de de la Terrasse

Québec, Canada G1V 0A6

e-mail : Normand.Teasdale@kin.ulaval.ca
Résumé Plusieurs études suggèrent que le vieillissement normal pourrait affecter la conduite automobile, particulièrement lors de la négociation d'intersections. Le but de cette étude naturalistique était de documenter les comportements aux intersections marquées par un panneau d'arrêt obligatoire et de contrôle de la vitesse chez des personnes âgées de 65 ans et plus. Vingt participants qui provenaient d'une région urbaine et d'une région semi-urbaine ont été sollicités pour une semaine d'expérimentation ( 5 hommes et 5 femmes de chaque région). L'activité de conduite était enregistrée à l'aide d'un GPS et des images du conducteur et de la route. L'analyse de l'ensemble des arrêts obligatoires (986) a montré que les freinages complets sont peu fréquents. Par exemple, la vitesse minimale observée pour plus de $60 \%$ des arrêts obligatoires était supérieure à $4,99 \mathrm{~km} / \mathrm{h}$ pour les hommes (38\% des arrêts pour les femmes). Les différences entre les régions au niveau des stratégies aux arrêts n'étaient pas significatives $(\mathrm{p}>0,05)$. Un total de 1561 excès de vitesse a été identifié. Un plus grand nombre de dépassements de vitesse a été observé dans des zones de limitation de vitesse à $50 \mathrm{~km} / \mathrm{h}$ (907 événements) $(\mathrm{p}<0,05)$. Parmi ces excès de vitesse, elles montrent des stratégies de conduite adoptées par le conducteur âgé en situations naturelles.

Mots clés âge $\cdot$ conduite automobile $\cdot$ Intersection $\cdot$ vitesse

Abstract Several studies suggest that normal ageing could affect the driving behavior, particularly when negotiating intersections. The purpose of the current naturalistic study was to document the behavior of drivers aged 65 and over at stop-controlled intersections and describe the way they deal with speed limits. Twenty participants were recruited for a 7-day experiment ( 5 men and 5 women from an urban and a semi-urban area). Displacements of the vehicle and 
images of the driver and scenery were recorded. The analysis of the stop-controlled intersections (986) showed that older drivers seldom stop completely at intersections. Overall, men showed a minimal speed that exceeded $4.99 \mathrm{~km} / \mathrm{h}$ for more than $60 \%$ of the intersections (38\% for the women). The braking strategies did not vary as a function of the area of residence $(p>.05)$. A total of 1561 speeding events were identified. Overall, speeding was more frequent on roads where the speed is limited to $50 \mathrm{~km} / \mathrm{h}$ (907 events) $(\mathrm{p}<.05)$. Of these events, 213 events showed a speed that was $20 \mathrm{~km} / \mathrm{h}$ above the speed limit. These observations are important because they show that some driving strategies are commonly used by elderly drivers (rolling stops and speeding).

\section{Keywords Age $\cdot$ Driving $\cdot$ Intersection $\cdot$ Speed}

\section{Introduction}

L'usage du véhicule personnel est plus qu'un moyen de se déplacer. Il fait partie du style de vie des sociétés modernes. À titre d'exemple, aux États-Unis, les personnes âgées de plus de 65 ans effectuent environ $90 \%$ de leurs déplacements en voiture dont $65 \%$ en tant que conducteurs [1]. De plus, la proportion de conducteurs âgés de plus de 65 ans dans les pays membres de l'« Organisation de coopération et de développement économiques » est en augmentation. Par exemple, au Québec, cette proportion représente actuellement $15 \%$ de l'ensemble des conducteurs et devrait atteindre plus de $25 \%$ des conducteurs en 2030 [2].

Certaines manœuvres de conduite, par exemple la négociation d'intersections marquées par un panneau d'arrêt obligatoire, semblent révéler des déficits liés au vieillissement normal. Par exemple, plusieurs études suggèrent que les intersections sont parmi les zones les plus risquées pour les personnes âgées [3]. En effet, comparativement aux conducteurs plus jeunes, les conducteurs âgés sont impliqués dans une plus grande proportion d'incidents de la route survenant aux intersections et lors de manœuvres de cédez-le-passage [4-9]. Par exemple, Preusser et al (1998) ont analysé le risque pour une personne âgée d'être impliquée dans une collision fatale à une intersection. Les données de collisions provenaient d'une base de données nationale aux États-Unis (Fatal Analysis Reporting System) et incluaient plus de 2500 collisions fatales pour des conducteurs de 40 à plus de 85 ans. Par rapport aux conducteurs âgés de 40 à 49 ans, ces auteurs ont observé que le risque d'être impliqué dans une collision impliquant plus d'un véhicule à une intersection marquée par un panneau d'arrêt obligatoire augmentait avec le vieillissement $(2,26$ fois plus élevé pour des conducteurs de 65 à 69 ans et jusqu'à 10,62 fois plus élevé pour des conducteurs âgés de plus de 85 ans). À partir de 70 ans, le risque était plus élevé même lorsque le conducteur ne modifiait pas sa trajectoire (c'est-à-dire aucun virage à gauche ou à droite).

Plus récemment, une étude comparative menée par Keay et al (2009) qui incluait 1425 conducteurs âgés entre 67 et 87 ans a montré que le comportement aux intersections était affecté par le lieu de résidence. En effet, dans cette étude, les conducteurs vivant en milieu rural n'ont pas effectué un arrêt complet à une intersection marquée par panneau d'arrêt obligatoire 1,7 fois plus souvent que les conducteurs vivant en milieu urbain. Selon les auteurs, ce résultat pourrait être attribué au nombre réduit d'usagers présents au moment du passage à l'intersection et à la meilleure visibilité aux intersections qui caractérisent les régions rurales. Ces conditions semblent faciliter l'intégration visuelle de la scène routière favorisant ainsi la prise de décision de ne pas freiner complètement aux intersections où l'arrêt pourrait sembler facultatif ( $p$. ex., aucun véhicule ou piéton, donc aucun risque de conflit possible) malgré la présence d'un panneau de signalisation prescrivant une immobilisation totale du véhicule avant de se réengager sur la route. Malheureusement, leurs données ne permettent pas de valider cette hypothèse puisque uniquement des données géospatiales étaient disponibles.

Le respect des limites de vitesse autorisées fait aussi partie des aptitudes et habitudes indispensables à une conduite automobile défensive (aussi appelée préventive) et des travaux récents montrent qu'il existe une relation entre le respect des limites de vitesse et les pratiques générales de sécurité routière (p. ex. l'établissement et l'application de limites de vitesse) [11]. De plus, le risque de collision est deux à trois fois plus élevé dans les zones limitées à 100 et $110 \mathrm{~km} / \mathrm{h}$ (sur des routes australiennes) que dans les zones de limitation à des vitesses inférieures [12]. Ce type de constat est informatif mais ne renseigne pas sur les habitudes de conduite. De fait, il est fort important de documenter la gestion de la vitesse sur les routes en situation réelle afin d'identifier les habitudes et les stratégies de gestion de la vitesse des conducteurs âgés. Ces informations sont essentielles afin de bonifier et mieux orienter les programmes offerts aux conducteurs qui désirent mettre à jour leurs connaissances sur la conduite défensive.

Le but de cette étude était donc de documenter, chez des participants de genre masculin et féminin âgés de 65 ans et plus, la négociation des intersections marquées par un panneau d'arrêt obligatoire et le contrôle de la vitesse en fonction des différentes limites de vitesse autorisées chez des conducteurs âgés en santé provenant d'une région urbaine (densité de population $>1000$ habitants/kilomètre ${ }^{2}$ ) ou d'une région semi-urbaine (densité de population près de 150 habitants/kilomètre ${ }^{2}$ ). 


\section{Méthodologie}

\section{Participants}

La population expérimentale de cette étude est constituée de vingt conducteurs âgés de 65 ans et plus. Dix participants (5 hommes et 5 femmes) provenaient de la ville de Québec (population d'environ 500000 habitants, densité de 1021 habitants/kilomètre ${ }^{2}$ ) et dix autres (5 hommes et 5 femmes) de municipalités régionales dans la région de Chaudière-Appalaches au sud de la ville de Québec. Plus spécifiquement, ces conducteurs provenaient des villes de Ste-Marie (40 $\mathrm{km}$ au sud de la ville de Québec, 13000 habitants, 120 habitants/kilomètre ${ }^{2}$ ) et de St-Georges de Beauce ( $85 \mathrm{~km}$ au sud de la ville de Québec, 30000 habitants, densité de 157 habitants/kilomètre ${ }^{2}$ ). Par convention, les termes régions urbaines (Québec) et semi-urbaines (Ste-Marie et St-Georges de Beauce) seront utilisés pour faire référence à ces deux régions. Le recrutement a été fait sur une base volontaire. Tous les conducteurs évalués devaient avoir une acuité visuelle conforme à la réglementation de la Société de l'Assurance Automobile du Québec (6/12 pour l'œil ayant la meilleure acuité visuelle), détenir un permis de conduire valide, conduire sa voiture un minimum de 3 fois par semaine et signer un formulaire de consentement éclairé. Ce protocole a préalablement été approuvé par le Comité d'éthique de la recherche de l'Université Laval.

Les individus sélectionnés ont complété un questionnaire permettant de documenter leurs habitudes de conduite (version française du Driving Habit Questionnaire) [13]. Des données sur la conduite étaient acquises automatiquement pendant 7 jours. Le Tableau 1 présente des informations sommaires sur les participants et les déplacements effectués au cours de l'étude. Une utilisation du véhicule est définie comme un trajet impliquant un démarrage et une mise à l'arrêt du moteur du véhicule. Aucune instruction spécifique sur la conduite n'était fournie aux participants. L'ensemble des participants a parcouru $2992,3 \mathrm{~km}$ au cours de 64,7 heures cumulées de conduite.

\section{Dispositif}

Le système d'acquisition des données, inspiré de celui utilisé par Lavallière et al (2012), était constitué de deux caméras USB (Logitech), d'un GPS (U-Blox) et d'un ordinateur portable. Une première caméra orientée vers l'extérieur permettait de filmer la route devant le véhicule. La deuxième caméra, orientée vers l'intérieur du véhicule permettait de filmer le conducteur (c'est-à-dire tête, cou, et tronc). Les deux caméras étaient maintenues en place par une ventouse fixée sur le pare-brise du véhicule, derrière le rétroviseur intérieur, donc à peine visible par le conducteur. Un logiciel développé par notre laboratoire permettait d'acquérir les images à une fréquence de $15 \mathrm{~Hz}(320$ pixels $\times 240$ pixels) et les données de position et de vitesse à une fréquence de $4 \mathrm{~Hz}$. Les images vidéo et les données géospatiales étaient horodatées avec une horloge commune. Les fonctionnalités d'alimentation et de mise en veille de l'ordinateur ont été modifiées de manière à permettre un retour de veille et un début d'acquisition des données avec le démarrage du véhicule. Un enregistrement des données était réalisé à toutes les 30 minutes ainsi qu'à la coupure du contact, soit à la fin d'un trajet. Ainsi, le système était autonome ; les participants n'avaient pas à se préoccuper de son état. L'ordinateur était fixé dans une boîte sécurisée dans le coffre arrière du véhicule. L'équipement était installé par l'expérimentateur dans le véhicule personnel du participant de manière à éviter les interférences possibles avec le champ visuel du conducteur ou de gêner l'utilisation habituelle du véhicule.

\section{Quantification et analyse des comportements de conduite}

Un logiciel de visualisation et de traitement des données a été développé à l'aide de l'environnement Matlab. Le logiciel permet d'afficher et de rejouer les images vidéo ainsi que les données de position et de vitesse simultanément. De même, les données de position peuvent

Tableau 1 Descriptif de l'utilisation du véhicule personnel au cours d'une semaine d'expérimentation

\begin{tabular}{|c|c|c|c|c|c|c|c|}
\hline \multirow[b]{2}{*}{ Milieu } & \multirow[b]{2}{*}{ Genre } & \multirow[b]{2}{*}{$\mathbf{n}$} & \multicolumn{2}{|l|}{ Âge (ans) } & \multirow[b]{2}{*}{$\begin{array}{l}\text { Utilisation moyenne } \\
\text { du véhicule }\end{array}$} & \multirow[b]{2}{*}{$\begin{array}{l}\text { Durée moyenne } \\
\text { de trajet (min) }\end{array}$} & \multirow[b]{2}{*}{$\begin{array}{l}\text { Distance moyenne } \\
\text { de trajet }(\mathbf{k m})\end{array}$} \\
\hline & & & minimum & maximum & & & \\
\hline \multirow[t]{2}{*}{ Semi-urbain } & Femme & 5 & 65 & 81 & 13 & 7,9 & 4 \\
\hline & Homme & 5 & 65 & 77 & 22 & 12,3 & 11,2 \\
\hline \multirow[t]{2}{*}{ Urbain } & Femme & 5 & 65 & 74 & 17 & 11,1 & 8,6 \\
\hline & Homme & 5 & 66 & 80 & 24 & 8,9 & 6,2 \\
\hline
\end{tabular}




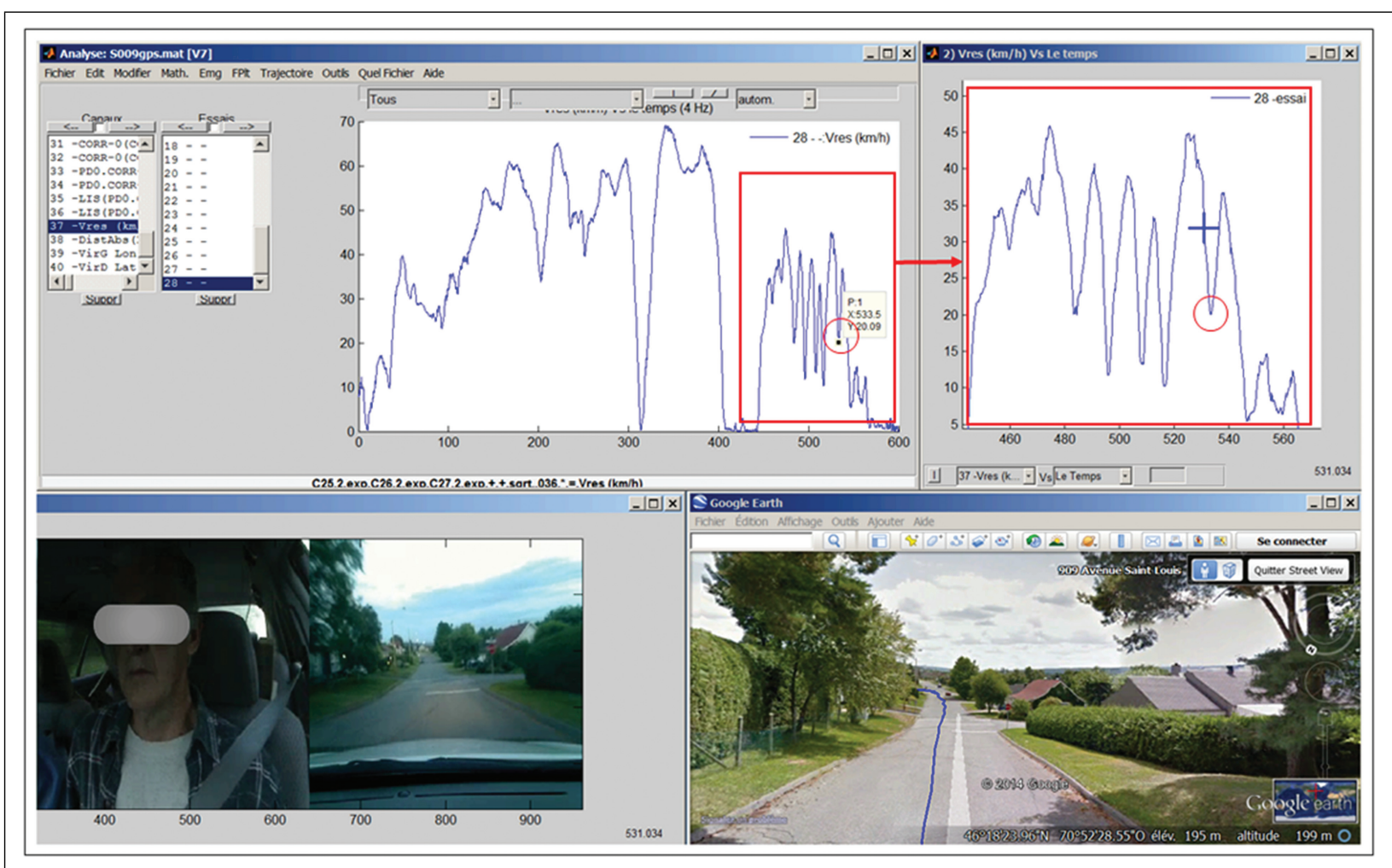

Fig. 1 llustration de l'interface développée sous Matlab qui permet de visualiser et d'analyser les données naturalistiques de conduite. Le panneau du haut à gauche illustre la vitesse du véhicule tout au long du trajet tandis que le panneau de droite présente un agrandissement de la section encadrée. Le curseur mobile $(+)$ représente la vitesse du véhicule synchronisée avec la vidéo du conducteur et de la scène (panneau du bas à gauche). Dans ce cas de figure, le suivi image par image de la séquence vidéo permet de détecter la vitesse minimale du véhicule lors de son passage (ou arrêt) au niveau de la ligne d'arrêt, qui était en l'occurrence légèrement supérieure à $20 \mathrm{~km} / \mathrm{h}$ (illustrée par les cercles sur les panneaux du haut). La vidéo permet aussi de détecter les mouvements de tête du conducteur (balayage visuel et angle mort). L'application SIG (en bas à droite) permet de positionner le parcours de conduite sur une carte interactive (simultanément avec les autres informations) et de retrouver/confirmer ainsi les informations nécessaires à la détection des arrêts aux intersections et des limitations de vitesse lorsque nécessaire

être présentées sur une carte à l'aide d'une application SIG (système d'information géographique). L'information ainsi disponible permet l'analyse et l'interprétation des caractéristiques particulières d'un trajet (limites de vitesse, présence d'intersections avec arrêt obligatoire...). Le logiciel permet aussi d'identifier des événements de conduite spécifiques en retraçant l'itinéraire emprunté (scène routière) et les comportements associés à la fois au conducteur (mouvements de la tête et des yeux) et au véhicule (position dans la voie, vitesse, le début et la fin d'une période de conduite en excès de vitesse...). La Figure 1 illustre une situation réelle de conduite impliquant un suivi chronologique de l'ensemble des événements rencontrés lors d'un trajet donné dans une perspective d'identification d'événements spécifiques (intersections avec arrêt obligatoire, dépassement de la limite de vitesse réglementaire) et de quantification des comportements de conduite respectifs.

\section{Arrêt aux intersections marquées par un panneau d'arrêt obligatoire}

Les intersections ont été identifiées à travers l'observation de la scène routière à l'aide de l'interface illustrée ci-dessus (Figure 1). Cette interface permet de visualiser simultanément la scène devant le véhicule, sa vitesse, les panneaux de circulation et d'identifier la présence ou non d'autres usagers de la route. Pour toutes les intersections avec arrêts obligatoires, la vitesse minimale, la trajectoire $\mathrm{du}$ véhicule suite au franchissement de la ligne d'arrêt obligatoire ainsi que la présence d'autres usagers de la route furent notées. Pour décrire les comportements d'arrêt, quatre classes de vitesse furent déterminées, soit lorsque la vitesse minimale atteinte était 1 . inférieure à $1 \mathrm{~km} / \mathrm{h}$, 2. entre 1 et $4,99 \mathrm{~km} / \mathrm{h}, 3$. entre 5 et $9,99 \mathrm{~km} / \mathrm{h}$, et 4 . supérieure à $10 \mathrm{~km} / \mathrm{h}$. Un arrêt était jugé complet si la vitesse minimale atteinte à la ligne d'arrêt était inférieure à $1 \mathrm{~km} / \mathrm{h}$ 
(première classe). Pour chacune des classes de vitesse et pour chacune des trajectoires du véhicule, un pourcentage d'intersections fut calculé pour chacun des participants (nombre d'intersections dans une classe/nombre total d'intersections pour le participant $\times 100$ ). Finalement, une codification binaire a été utilisée pour documenter la présence d'autres usagers de la route au niveau de l'intersection (aucun autre usager et présence d'autres usagers sans égard au fait que ces autres usagers puissent être d'autres véhicules, des piétons ou des cyclistes).

\section{Contrôle de la vitesse de conduite}

La vitesse du véhicule était obtenue à partir des données enregistrées par le GPS. Les zones de limitation de vitesse (début et fin des zones de 30, 50, 70, 80, 90 et $100 \mathrm{~km} / \mathrm{h}$ ) furent identifiées manuellement pour chacun des trajets de chacun des participants à l'aide du SIG et des images vidéo. La période de temps pendant laquelle la vitesse du véhicule était supérieure à celle autorisée ainsi que la vitesse maximale atteinte pendant chacune de ces périodes furent également notées.

\section{Résultats}

\section{Arrêt aux intersections marquées par un panneau d'arrêt obligatoire}

Au total, 986 intersections avec arrêts obligatoires furent notées. Le nombre d'intersections était légèrement inférieur pour les participants provenant de la région semi-urbaine que pour les participants provenant de la région urbaine mais cette différence n'était pas significative (420 en région semi-urbaine vs 566 en région urbaine ; $\mathrm{t}(18)=1,17$, $p>0,05)$. Les participants de genre féminin ont franchi un plus grand nombre d'intersections avec arrêts obligatoires que les participants de genre masculin (571 vs 415 intersections, respectivement, $\mathrm{t}(18)=6,15, \mathrm{p}<0,05)$. Au-delà de ces résultats descriptifs, la vitesse minimale atteinte lors d'un arrêt obligatoire a été quantifiée. Une première question importante concernait l'effet du genre $\mathrm{du}$ conducteur et de sa région d'appartenance sur son comportement de freinage. Compte tenu du petit nombre de participants, les pourcentages obtenus pour les différentes classes de vitesse furent soumis à des ANOVAs indépendantes Régions $\times$ Classes de Vitesse et Genre $\times$ Classes de Vitesse avec mesures répétées sur le facteur Vitesse. L'ANOVA Régions $\times$ Classes de Vitesse n'a montré aucune différence significative entre les stratégies de freinage des conducteurs provenant de la région urbaine et des conducteurs provenant de la région semi-urbaine $(p>0,05)$. Les résultats de l'ANOVA Genre $\times$ Classes de

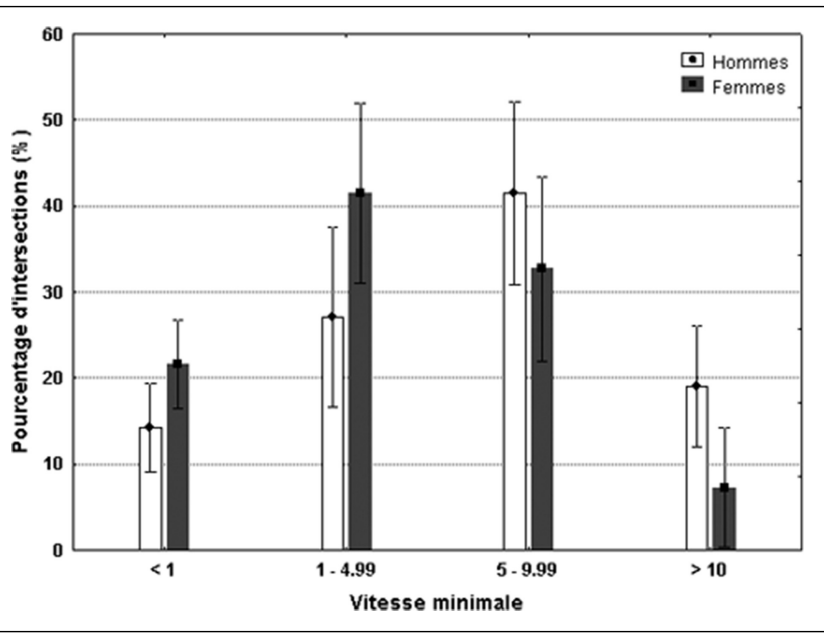

Fig. 2 Représentation graphique du pourcentage d'intersections rencontrées par les participantes et les participants en fonction de quatre classes de vitesse minimale observées lors de passages à des intersections avec un arrêt obligatoire

Vitesse ont montré un effet significatif du facteur Classes de Vitesse $(F(3,54)=13,2, p<0,001)$ ainsi qu'une interaction significative Genre $\times$ Classes de Vitesse $(F(3,54)=3,6$, $p<0,05$, Figure 2). Une première observation est que les hommes et les femmes montrent des variations importantes dans le comportement de freinage aux arrêts obligatoires. En effet, un arrêt complet est observé pour $21 \%$ des arrêts obligatoires pour les femmes et pour $14 \%$ des arrêts obligatoires pour les hommes. D'autre part, les femmes adoptent un comportement de freinage plus conservateur puisque, pour plus de $60 \%$ des intersections, leur vitesse maximale de passage à la ligne d'arrêt est inférieure à $5 \mathrm{~km} / \mathrm{h}(21 \%$ des arrêts obligatoires avec une vitesse inférieure à $1 \mathrm{~km} / \mathrm{h}$ et $41 \%$ des arrêts obligatoires avec une vitesse entre 1 et $4,99 \mathrm{~km} / \mathrm{h}$ ). Cette tendance est inversée chez les hommes, lesquels montrent une vitesse minimale de passage à la ligne d'arrêt supérieure à $5 \mathrm{~km} / \mathrm{h}$ pour plus de $60 \%$ des intersections obligatoires $(41 \%$ des arrêts obligatoires avec une vitesse entre 5 et $9,99 \mathrm{~km} / \mathrm{h}$ et $19 \%$ des arrêts obligatoires avec une vitesse supérieure à $10 \mathrm{~km} / \mathrm{h}$ ).

Une analyse complémentaire en fonction de la présence ou non d'autres usagers de la route lors du passage à l'intersection a été réalisée (sans égard à la région ni au genre des participants). La Figure 3a présente pour les intersections libres de tout autre usager de la route (au total, 448 intersections), les moyennes de pourcentages d'intersections pour chacune des classes de vitesse. Les pourcentages obtenus pour chacun des participants furent soumis à une ANOVA à mesures répétées à un facteur (Classes de Vitesse). L'ANOVA montre un effet significatif $(\mathrm{F}(3,57)=$ $13,28, \mathrm{p}<0,001)$. Il est possible d'observer que les arrêts complets (vitesse minimale $<1 \mathrm{~km} / \mathrm{h}$ ) ne représentent que 


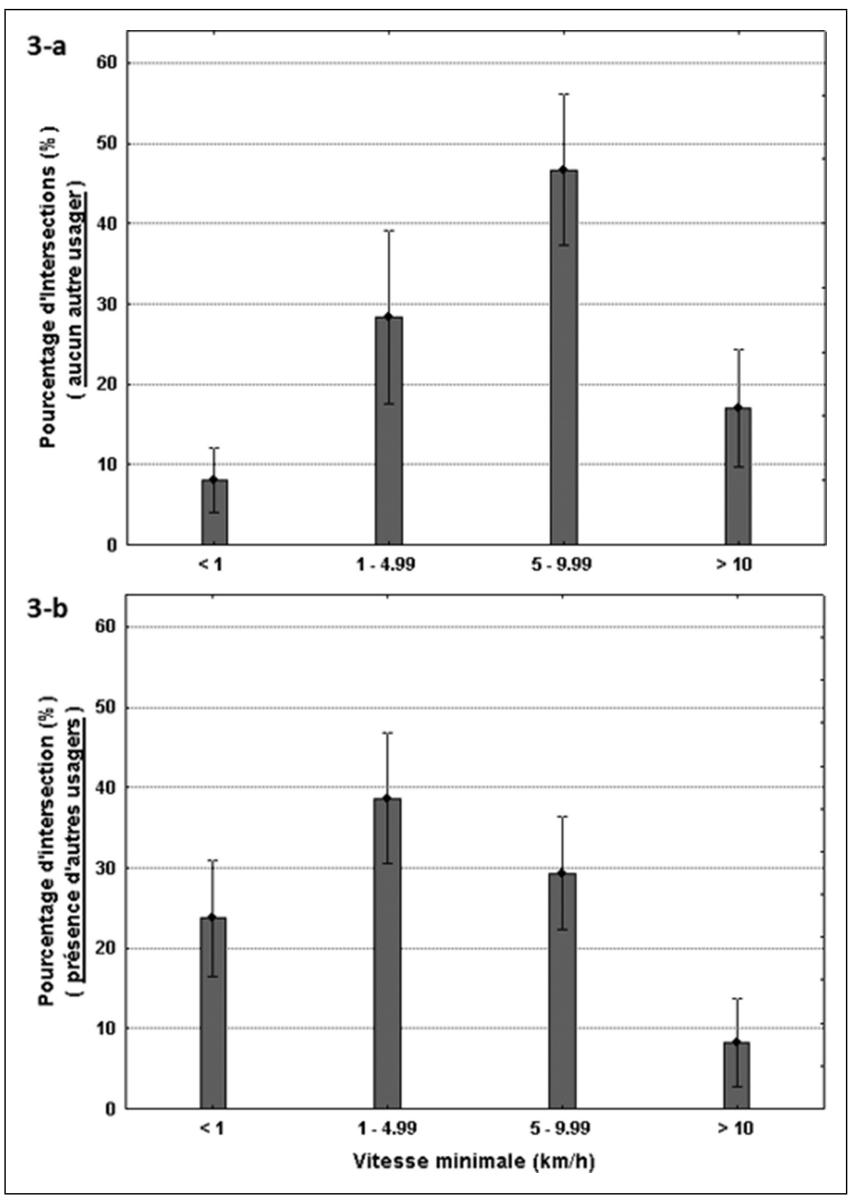

Fig. 3 Représentation graphique du pourcentage d'intersections en fonction des vitesses minimales observées lors du passage à des intersections avec un arrêt obligatoire. La Figure 3a illustre les pourcentages d'intersections où aucun autre usager de la route n'est visible tandis que la Figure $3 \mathrm{~b}$ représente les pourcentages d'intersections lorsque celle-ci était partagée avec un ou plusieurs autres usagers de la route

$8 \%$ des intersections. La vitesse était supérieure à $4,99 \mathrm{~km} / \mathrm{h}$ pour plus de $60 \%$ des intersections (la vitesse était entre 5 et $9,99 \mathrm{~km} / \mathrm{h}$ pour $46 \%$ des intersections et supérieure à $10 \mathrm{~km} / \mathrm{h}$ pour $17 \%$ des intersections). Une analyse post-hoc (Bonferroni) montre que le pourcentage d'intersections avec un arrêt complet (vitesse $<$ à $1 \mathrm{~km} / \mathrm{h}$ ) était inférieur aux pourcentages observés pour les classes de vitesse allant de 1 à $4,99 \mathrm{~km} / \mathrm{h}$ et de 5 à $9,99 \mathrm{~km} / \mathrm{h}(\mathrm{p}<0,05)$. Ce constat montre que, pour une très forte proportion des intersections, les conducteurs âgés évalués dans cette étude ont l'habitude de ne pas freiner complètement leur véhicule lorsqu'aucun autre usager de la route n'est à proximité de l'intersection.

La Figure $3 \mathrm{~b}$ illustre les pourcentages d'intersections pour chacune des classes de vitesse lorsqu'un autre usager de la route était visible lors du passage à l'intersection (au total, 538 intersections). Les pourcentages obtenus pour chacun des participants furent aussi soumis à une ANOVA à mesures répétées à un facteur (Classes de Vitesse). L'ANOVA montre un effet significatif $(\mathrm{F}(3,57)=10,73$, $\mathrm{p}<0,001)$. Dans cette situation, on peut noter que les conducteurs ont freiné complètement (vitesse $<$ à $1 \mathrm{~km} / \mathrm{h}$ ) pour près de $24 \%$ des intersections. Au total, plus de $60 \%$ des intersections montraient une vitesse inférieure à $5 \mathrm{~km} / \mathrm{h}$ (la vitesse était inférieure à $1 \mathrm{~km} / \mathrm{h}$ pour $24 \%$ des intersections et entre 1 et $4,99 \mathrm{~km} / \mathrm{h}$ pour $38,3 \%$ des intersections). À l'inverse, le pourcentage d'intersections montrant un freinage à des vitesses supérieures à $9,99 \mathrm{~km} / \mathrm{h}$ était plus faible que les pourcentages d'intersections pour les trois autres catégories de vitesse inférieures ( $8 \%$ des intersections avec présence d'usagers). Une analyse post-hoc (Bonferroni) montre un effet significatif pour les 3 comparaisons $(\mathrm{p}<0,05)$. Étonnamment, malgré la présence d'autres usagers de la route, on observe des pourcentages élevés d'intersections où un arrêt complet n'est pas observé. Malgré un plus faible pourcentage d'intersections montrant une vitesse minimale de passage supérieure à $9,99 \mathrm{~km} / \mathrm{h}$, ces résultats suggèrent un comportement d'ajustement du freinage en fonction de la présence d'autres usagers de la route et d'une analyse du risque de collision associé à la situation. Ainsi, le conducteur freinerait complètement lorsqu'il détecte la présence d'un autre usager de la route. Toutefois, il ajusterait sa stratégie et cesserait de freiner complètement lorsqu'il estime qu'aucun risque n'est encouru.

\section{Contrôle de la vitesse de conduite}

Un deuxième objectif de cette étude était de documenter le comportement des conducteurs âgés face aux limitations de vitesse. Le nombre total d'excès de vitesse identifiés s'élève à 1561 événements répartis également entre les participants de la région urbaine et ceux de la région semi-urbaine (851 et 710 excès de vitesse, respectivement $; \mathrm{t}(18)=0,52$, $p>0,05)$. Le nombre moyen d'excès de vitesse ainsi que leur durée moyenne (en pourcentage de la durée de chacun des trajets) pour l'ensemble des participants répartis selon le genre et la région de résidence sont présentés au Tableau 2.

Pour les deux régions de résidence, le nombre d'excès de vitesse des hommes était supérieur à celui des femmes (en moyenne par conducteur, 105 vs. 50 excès de vitesse, respectivement ; $\mathrm{t}(18)=1,94, \mathrm{p}<0,05)$. Pour chacun des excès de vitesse observés, la durée de dépassement de la limite de vitesse autorisée et la vitesse maximale observée ont été identifiées. La Figure 4 illustre, pour les hommes et les femmes, la répartition des excès de vitesse (en pourcentage du nombre d'excès de vitesse par genre) en fonction des six zones de vitesse (c'est-à-dire, 30, 50, 70, 80, 90 et $100 \mathrm{~km} / \mathrm{h}$ ). Les résultats furent soumis à une ANOVA 
Tableau 2 Descriptif des excès de vitesse commis au cours d'une semaine de conduite

\begin{tabular}{lllll}
\hline Milieu & Genre & $\mathbf{n}$ & Nombre moyen d'excès & Durée d'excès de vitesse/Durée de trajet (\%) \\
\hline Semi-urbain & Femme & 5 & 32 & 12,6 \\
\multirow{3}{*}{ Urbain } & Homme & 5 & 110 & 31,3 \\
& Femme & 5 & 68 & 17,9 \\
& Homme & 5 & 102,2 & 24,7 \\
\hline
\end{tabular}

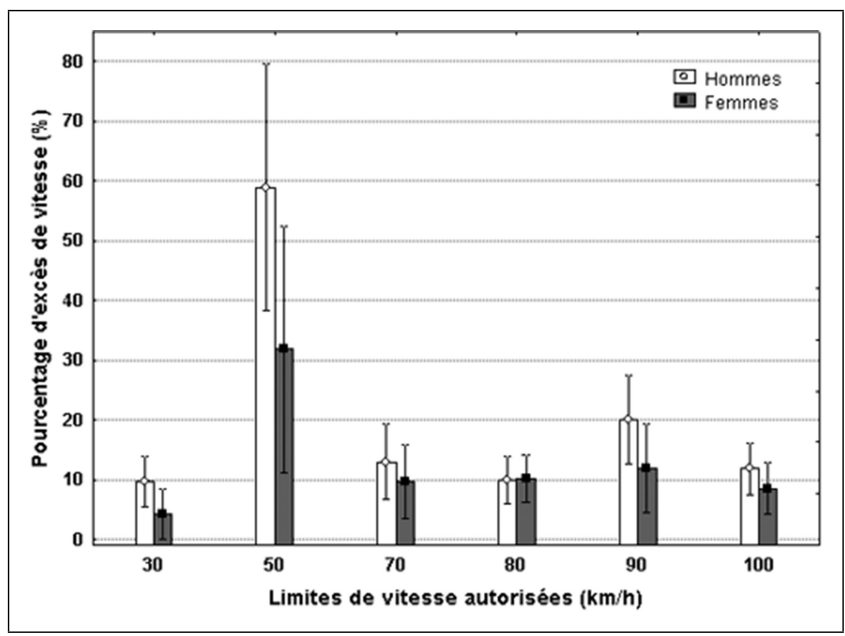

Fig. 4 Représentation graphique des pourcentages d'excès de vitesse de l'ensemble des participants en fonction des limites de vitesse permises

Limites de Vitesse $\times$ Genre. L'ANOVA montre un effet significatif du facteur Limites de Vitesse $(F(5,90)=24,88$, $\mathrm{p}<0.001$ ). Une analyse post-hoc (Bonferroni) montre que la majorité des dépassements de vitesse observés se produit dans des zones de limitation de vitesse à $50 \mathrm{~km} / \mathrm{h}$ aussi bien chez les hommes que chez les femmes (environ $45 \%$ pour l'ensemble des conducteurs ; $p<0,05$ pour toutes les comparaisons avec la zone de $50 \mathrm{~km} / \mathrm{h}$ ).

Il est important de mentionner que le nombre d'excès de vitesse n'était pas réparti également entre les conducteurs et les zones de limites de vitesse. Par exemple, alors que tous les conducteurs ont commis des excès de vitesse pour les zones de 30 et $50 \mathrm{~km} / \mathrm{h}$, uniquement 9 conducteurs ont conduit dans des zones de $100 \mathrm{~km} / \mathrm{h}$. Afin de catégoriser ces événements pour chacune des zones de limite de vitesse, trois paliers d'excès de vitesse ont été choisis arbitrairement : 1 . inférieur à $10 \mathrm{~km} / \mathrm{h}, 2$. entre 10 et $20 \mathrm{~km} / \mathrm{h}$ et 3 . au-dessus de $20 \mathrm{~km} / \mathrm{h}$. Le Tableau 3 présente le nombre d'excès de vitesse commis par les conductrices et les conducteurs pour chacune des zones de limites de vitesse. Plus de la moitié des excès de vitesse commis par les conductrices et les conducteurs étaient inférieurs à
$10 \mathrm{~km} / \mathrm{h}(66,6$ et $53 \%$ des excès de vitesse des femmes et des hommes, respectivement). D'autre part, près d'un tiers de l'ensemble des excès de vitesse était entre 10 et $20 \mathrm{~km} / \mathrm{h}(27 \%$ et $29 \%$, respectivement). Finalement, les femmes ont commis 31 excès de vitesse supérieurs à $20 \mathrm{~km} / \mathrm{h}$ alors que les hommes en ont commis 182 ( $6 \%$ et $17 \%$, respectivement). Étonnamment, 514 excès de vitesse furent observés pour des zones de limite de vitesse de $50 \mathrm{~km} / \mathrm{h}$. La Figure 5 illustre la répartition des excès par zone de vitesse chez les participantes et les participants. Chacun des points représente un excès de vitesse. Cette figure permet d'apprécier la dispersion des événements d'excès de vitesse, les valeurs maximales d'excès de vitesse ainsi que les durées des excès (panneau du bas). Il est possible de noter quelques événements montrant des vitesses largement supérieures aux limites de vitesse, et ce principalement lorsque les conducteurs conduisaient sur des routes limitées à $50 \mathrm{~km} / \mathrm{h}$. L'hétérogénéité des observations entre les participants ne permettait pas de réaliser des analyses statistiques inférentielles.

\section{Discussion}

Les résultats obtenus lors de cette étude naturalistique montrent que l'arrêt à une intersection est souvent

Tableau 3 Répartition des excès de vitesse par limites de vitesse permise et par paliers d'excès chez les participantes et les participants

\begin{tabular}{|llllllll}
\hline & & \multicolumn{7}{c}{ Limites de vitesse } \\
\cline { 3 - 8 } & Excès de vitesse & $\mathbf{3 0}$ & $\mathbf{5 0}$ & $\mathbf{7 0}$ & $\mathbf{8 0}$ & $\mathbf{9 0}$ & $\mathbf{1 0 0}$ \\
\hline Femmes & $0<$ vitesse $\geq 10$ & 14 & 213 & 35 & 19 & 33 & 21 \\
& $10<$ vitesse $\geq 20$ & 18 & 88 & 11 & 11 & 6 & 3 \\
Hommes & vitesse $>20$ & 8 & 17 & 5 & 1 & 0 & 0 \\
& $0<$ vitesse $\geq 10$ & 33 & 301 & 44 & 52 & 99 & 38 \\
& $10<$ vitesse $\geq 20$ & 37 & 182 & 33 & 15 & 33 & 19 \\
& vitesse $>20$ & 13 & 106 & 30 & 2 & 21 & 10 \\
\hline
\end{tabular}




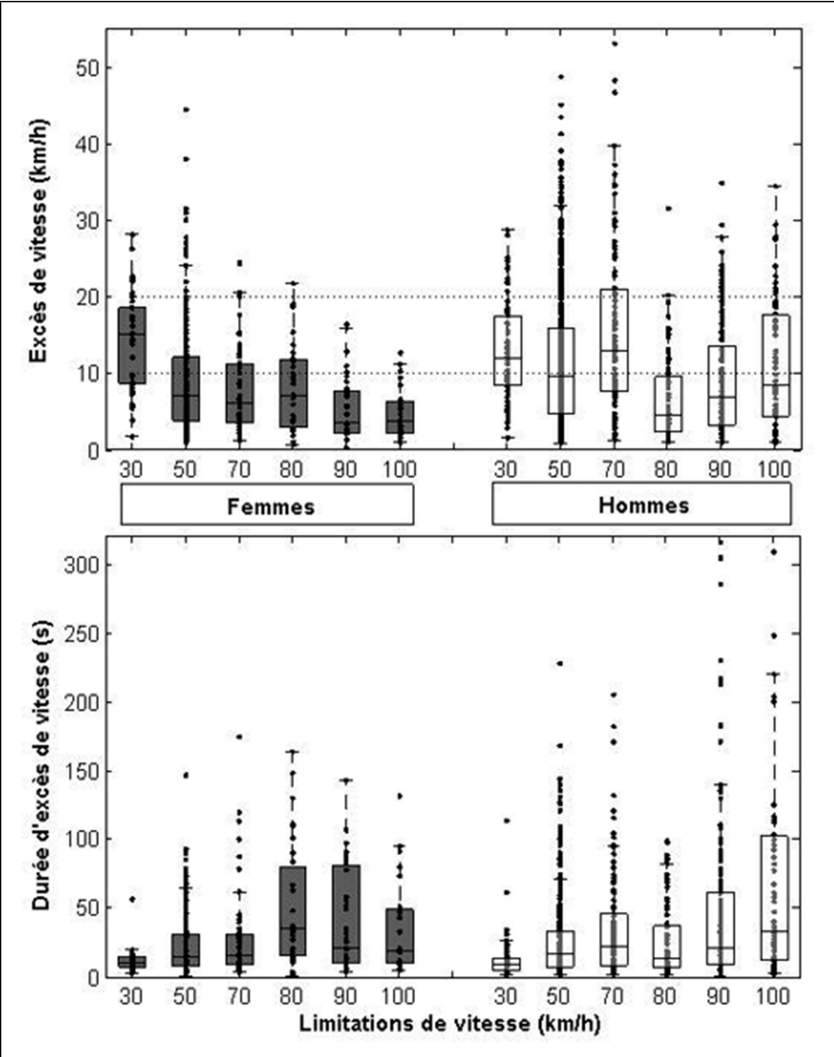

Fig. 5 Représentation graphique des valeurs maximales d'excès de vitesse (en $\mathrm{km} / \mathrm{h}$, panneau du haut) et de leur durée respective (en sec, panneau du bas) en fonction des limites de vitesse autorisées chez les femmes et les hommes. Pour chacune des catégories de limites de vitesse, un point représente un excès de vitesse. Les résultats répartis par genre pour chaque catégorie de limite de vitesse et chaque variable sont présentés sous forme de boîte à moustaches. La boîte inclut les données du premier au troisième quartile (c'est-à-dire de 25 à $75 \%$ des observations). La valeur médiane du nuage de points est représentée par une barre horizontale au milieu de la boîte à moustaches. Les extrémités des moustaches représentent 1,5 fois la valeur de l'écart interquartile. Les points situés au-dessus de la moustache supérieure sont des valeurs extrêmes

incomplet chez les conducteurs âgés en santé. Ce comportement, quoique légèrement moins fréquent pour les conductrices que pour les conducteurs, semble généralisé peu importe la région de provenance du conducteur. Ce comportement pourrait résulter d'une stratégie de prise de risque calculé. Cette explication est en accord avec la théorie de l'homéostasie du risque de Wilde (1989) (risk homeostasis theory) suggérant que les conducteurs adaptent leurs comportements en fonction du risque qu'ils perçoivent pour une situation donnée et du niveau de risque qu'ils sont prêts à accepter. Wilde (1989) a noté à travers une revue de la littérature que des modifications de la signalisation routière, voire ici une diminution de la vitesse maximale permise, n'ont pas toujours permis de réduire le nombre de collisions. D'autres facteurs pourraient donc déterminer le risque de collisions à une intersection. Dans le cas de la présente étude, le comportement du conducteur aux intersections est abordé en termes de facteurs déterminants de la prise de décision, soit d'effectuer un arrêt complet plutôt que de risquer une collision. Ces facteurs furent répartis en deux catégories. On distingue des facteurs liés au conducteur lui-même, c'est-à-dire son genre et sa région de résidence, et d'autres facteurs en lien avec la densité de la circulation, à savoir la présence ou non d'usagers de la route dans le champ visuel du conducteur lorsqu'il s'approche de l'intersection. Les résultats obtenus ne permettent pas d'établir des différences entre les conducteurs âgés des régions urbaines et ceux des régions semi-urbaines, contrairement à l'étude de Keay et al (2009) qui montre que les conducteurs ruraux font plus d'arrêts incomplets que les conducteurs urbains. Cependant, des tendances ont été identifiées selon le genre $\mathrm{du}$ conducteur. Le nombre de freinages complets aux intersections était faible pour l'ensemble des participants (moins de $20 \%$ ). Par contre, les femmes avaient tendance à ralentir plus souvent à des vitesses faibles se rapprochant des arrêts complets à l'inverse des conducteurs masculins qui manifestaient un comportement moins conservateur. Même si aucune omission de panneau d'arrêt n'a été observée (c'est-à-dire, aucun freinage ou ralentissement à l'approche d'un arrêt obligatoire), plusieurs intersections ont été traversées sans qu'un arrêt complet ne soit effectué et plusieurs lignes d'arrêt ont été franchies à des vitesses minimales supérieures à $20 \mathrm{~km} / \mathrm{h}$ (Figure 1). Des résultats comparables ont été obtenus par Teasdale et al (2011) qui ont étudié le comportement de conduite de personnes âgées dans des conditions réelles sur un parcours de $12 \mathrm{~km}$ avec 9 intersections ayant une signalisation par panneau d'arrêt. Ces auteurs ont rapporté des vitesses minimales de passage à l'intersection allant jusqu'à $19 \mathrm{~km} / \mathrm{h}$ et que les conducteurs âgés arrêtaient complètement leur véhicule dans moins de $10 \%$ des intersections. Dans notre étude, la compréhension de cette variabilité de comportement de freinage a été facilitée par l'étude des conditions de la circulation environnante et l'analyse de leurs effets sur la qualité des arrêts. Sans prétendre que nos résultats soient spécifiques aux aînés, notre étude dégage une tendance générale : les participants prennent fréquemment le risque de franchir la ligne d'arrêt à basse vitesse si l'intersection est libre, toutefois, ils freinent plus si les conditions de la circulation à l'intersection l'imposent. En plus d'être proscrite par la loi, cette mauvaise habitude de conduite présente un risque élevé pour la sécurité routière car le temps nécessaire pour éviter une collision possible lors de l'arrivée d'un autre usager de la route ou d'une détection 
tardive de celle-ci est réduit. Les personnes âgées sont en particulier plus vulnérables lors de tels événements car on observe généralement un ralentissement de la vitesse de traitement de l'information [17] et une réaction plus lente aux événements imprévus [18]. De plus, les conducteurs qui n'arrêtent pas complètement leur véhicule à une intersection risquent de réduire leur champ visuel utile et de ne pas percevoir les autres usagers. C'est d'ailleurs un des facteurs fréquemment rapportés lors d'une collision routière (looked but failed to see). Plusieurs conducteurs impliqués dans une collision voient apparaître les autres usagers soudainement ou prétendent avoir regardé sans jamais avoir vu l'autre usager de la route. Hakamies-Blomqvist (1993) a rapporté que $43 \%$ de conducteurs âgés de 65 ans et plus n'étaient même pas conscients du danger avant la collision, contre $26 \%$ chez des conducteurs plus jeunes (26 à 40 ans). La compréhension de ce phénomène va au-delà de l'analyse des comportements du conducteur lui-même et nécessite aussi une exploration des éléments de l'environnement de conduite qui pourraient contraindre l'ensemble des usagers de la route (et non pas uniquement le conducteur âgé) à respecter les arrêts obligatoires aux intersections. Bien qu'elle soit une approche contre intuitive à ce qui est actuellement préconisé dans le développement d'infrastructure routière, c'est peut-être en diminuant la visibilité que l'on pourrait forcer les conducteurs à effectuer un arrêt complet aux intersections et non pas en favorisant l'exploration visuelle sur une grande étendue avant l'arrivée à l'intersection. Ainsi, le dégagement du champ de vision aux intersections aurait pour effet délétère que le conducteur augmente sa prise de risque ; il n'immobilise pas complètement son véhicule lorsqu'il perçoit que l'intersection est libre (c'est-à-dire absence d'autres véhicules ou piétons ou non perception de ceux-ci).

La gestion de la vitesse de conduite dépasse le simple fait de respecter les limites de vitesse autorisées par le code de la route. Conduire à vitesse rapide ou au-delà des limites autorisées réduit la qualité des informations visuelles et raccourcit le temps où une manœuvre d'évitement sécuritaire peut être effectuée. Le risque de privation d'informations indispensables à la conduite automobile est élevé chez les conducteurs âgés en particulier car leur capacité à détecter les informations visuelles disponibles sur la scène routière est réduite $[19,20]$. D'après les études de conduite menées en simulateur par Rogé et al (2003, 2004), les conducteurs âgés détectent moins d'informations périphériques par rapport à une cible centrale que les jeunes (cible : véhicule qui précède sur la même voie). Ce rétrécissement du champ visuel utile induit un phénomène de vision en tunnel [21-23]. L'excès de vitesse serait donc néfaste à la qualité de conduite des aînés d'autant plus que leur capacité à réagir rapidement à d'éventuels événements imprévisibles est réduite [18]. Le constat est que nos participants ont montré des habitudes de contrôle de la vitesse à risque et conduisent souvent au-dessus des limites autorisées $(12 \%$ à $32 \%$ des durées totales de trajet, Tableau 2).

Dans l'ensemble, les excès de vitesse étaient plus élevés dans les zones correspondant aux limites de vitesse basses qui pourraient souvent correspondre à des portions de route dans des zones scolaires ou des quartiers résidentiels (30 et $50 \mathrm{~km} / \mathrm{h}$, respectivement). L'une des explications possibles de ce phénomène serait de penser au temps nécessaire pour traiter la quantité d'informations visuelles nécessaire à la conduite. Ainsi, la régulation de la vitesse serait tributaire de la quantité d'informations à traiter. L'indifférence des conducteurs à l'égard de la vitesse lorsqu'ils conduisaient dans des zones de faibles vitesses pourrait alors être expliquée par le fait que les conditions routières présentaient une charge attentionnelle faible relativement au temps de traitement requis. Notre observation qualitative des trajets effectués par les personnes âgées a permis d'identifier certains écarts de conduite qui peuvent témoigner du faible niveau d'exigence attentionnelle sur certaines portions de route à basse vitesse où les conducteurs respectaient les limites de vitesse. À titre d'exemple, l'attention de certains conducteurs était volontairement détournée vers les scènes latérales (paysage, trottoirs, commerces...) ou vers l'utilisation de leur téléphone cellulaire alors qu'ils conduisaient à basse vitesse sur des portions de route limitées à 30 et $50 \mathrm{~km} / \mathrm{h}$. Sans généraliser ces comportements inappropriés à l'ensemble des participants, la mauvaise gestion de la vitesse dans les zones avec limites de vitesse basses semble se manifester sous forme de deux profils : 1. conduire au-dessus de la vitesse permise, 2 . conduire à la vitesse réglementaire en associant une autre tâche à risque.

D'un autre point de vue, les zones de vitesse ont tendance à affecter les amplitudes de dépassement des limites permises. Les conducteurs évalués étaient de plus en plus conservateurs avec l'augmentation des limitations de vitesse, ce qui pourrait être dû à la contrainte des vitesses qui sont déjà élevées avant même de commettre des excès. En effet, il a été démontré que les conducteurs détectent moins d'informations disponibles dans leur champ visuel quand ils conduisent à haute vitesse $[19,20]$. Cependant, la fréquence notable de dépassements excessifs de la vitesse permise dans des zones de 90 et $100 \mathrm{~km} / \mathrm{h}$ laisse penser que certains conducteurs âgés négligent souvent les vitesses qu'ils devraient respecter même sur les routes à haute vitesse. L'une des incitations au respect des limites de vitesse serait de répéter plus fréquemment les panneaux de limitation de vitesse et de présenter des messages de prévention routière afin de le rappeler aux usagers imprudents ou peu attentifs.

Ce travail comporte un certain nombre de limitations. Clairement, la lourdeur du protocole fait en sorte que 
la taille de notre échantillonnage est petite. De plus, nous avons obtenu un échantillonnage de convenance et sa représentativité peut-être questionnée. Il importe donc de ne pas généraliser les observations à l'ensemble des conducteurs âgés. Nous avons exclu les conducteurs ayant un problème de santé et il est probable que notre échantillonnage inclue peu de conducteurs craintifs d'être évalués. D'autre part, il est intéressant d'observer qu'une étude récente avec des conducteurs souffrant de la maladie de Parkinson [24] confirme que des excès de vitesse sont aussi observés pour ces personnes de même que pour un groupe de conducteurs âgés sans problème de santé. Dans notre étude naturalistique, les vitesses moyennes sur des routes urbaines et rurales, de même que sur les autoroutes sont toutes supérieures aux limites de vitesse affichées et les valeurs moyennes présentées s'apparentent à celles observées dans l'étude de Crizzle et Myers [24] . Même si ce travail n'offre pas de comparaison directe avec d'autres groupes de conducteurs, il est probable que des observations similaires puissent être faites avec des conducteurs plus jeunes. Une telle observation soulèverait la possibilité que ces stratégies sont acquises et reflètent simplement la continuité des pratiques de conduite. Ainsi, plusieurs questions demeurent. Est-ce des stratégies accidentogènes? Si c'est le cas, est-il possible de modifier ces stratégies et de quantifier leurs effets sur la présence de collisions ? Une réponse à ces questions est importante mais dépasse largement le cadre de notre travail.

En conclusion, le caractère naturalistique de notre travail de recherche a permis d'évaluer de manière objective les comportements de conducteurs âgés participants à l'étude sur des routes qu'ils empruntent habituellement. Même si les parcours de conduite étaient enregistrés sur seulement 7 jours, les informations sur les habitudes de négociation des intersections et de respect des limites de vitesse semblent traduire la réalité des comportements de conduite des personnes âgées. Les conducteurs âgés de 65 ans et plus ont clairement tendance à conduire en fonction d'une perception du risque. Cette observation ne vise pas à ostraciser le conducteur âgé puisque dans le meilleur des cas, et comme nous mentionnions ci-dessus, des comportements similaires sont fort probablement présents chez des conducteurs plus jeunes.

L'automobile, pour plusieurs personnes âgées, leur permet de maintenir une autonomie fonctionnelle et parfois même celle de leur entourage. Une augmentation des dépressions et d'un sentiment d'isolement a d'ailleurs été observée chez les personnes âgées à qui le permis de conduire avait été enlevé $[25,26]$. Il importe donc de mettre en place des programmes de formation qui vont permettre aux conducteurs âgés de favoriser le maintien d'une conduite défensive afin d'éviter la perte du permis de conduire. Conduire est une tâche complexe et les causes d'une collision sont souvent multifactorielles. Cependant, plusieurs travaux ont mis en évidence des taux de collision plus importants aux intersections (p. ex., Preusser et al, 1998). Une conduite préventive et défensive basée sur le respect des réglementations mises en place pourrait contribuer à diminuer ces taux.

\section{Remerciements}

Cette recherche était supportée par un financement du Programme de recherche sécurité routière FRQNT-MTQFRQS. Des travaux antérieurs réalisés dans le cadre de subventions obtenues avec le Centre d'excellence AUTO21 ont permis la réalisation de ce projet. Un remerciement particulier à tous les participants pour leur précieuse collaboration ainsi qu'à Mikael Quirion pour sa collaboration lors de l'acquisition d'une partie des données.

\section{Références}

1. Granda TM, Thompson S (2006) The older driver comes of age. Public Roads 69(4). Disponible sur : http://www.fhwa.dot.gov/publications/publicroads/06jan/04.cfm (consulté en janvier 2014).

2. Société de l'Assurance Automobile du Québec (2010) Dossier Statistique : Bilan 2009. Disponible sur : http://www.saaq.gouv.qc.ca/rdsr/sites/files/12009002.pdf (consulté en janvier 2014).

3. Canada Transport (2013) A Quick Look at Intersection Crashes in Canada. Disponible sur : http://www.tc.gc.ca/eng/motorvehiclesafety/tp-tp2436-rs200806menu-839.htm (consulté en février 2014).

4. Garber NJ, Srinivasan R (1991a) Characteristics of accidents involving elderly drivers at intersections. Transport Res 1325: $8-16$.

5. Garber NJ, Srinivasan R (1991b) Risk assessment of elderly drivers at intersections: Statistical modeling. Transport Res 1325: $17-22$.

6. Preusser DF, Williams AF, Ferguson SA et al. (1998) Fatal crash risk for older drivers at intersections. Accid Anal Prev 30(2): 151159.

7. Hakamies-Blomqvist L (1993) Fatal accidents of older drivers. Accid Anal Prev 25(1): 19-27.

8. Hakamies-Blomqvist L (1994) Aging and fatal accidents in male and female drivers. J Gerontol 49(6): 286-290.

9. Langford J, Koppel S, Andrea D, Fildes B (2006) Determining older driver crash responsibility from police and insurance data. Traffic Inj Prev 7(4): 343-351.

10. Keay L, Jasti S, Munoz B et al (2009) Urban and rural differences in older drivers' failure to stop at stop signs. Accid Anal Prev 41(5): 995-1000.

11. Amador L, Willis CJ (2014) Demonstrating a correlation between the maturity of road safety practices and road safety incidents. Traffic Inj Prev 15(6): 591-7.

12. Thompson JP, Baldock MRJ, Mathias JL, Wundersitz LN (2013) An examination of the environmental, driver and vehicle factors associated with the serious and fatal crashes of older rural drivers. Accid Anal Prev 50: 768-775. 
13. Owsley C, Stalvey B, Wells J, Sloane ME (1999) Older drivers and cataract: driving habits and crash risk. J Gerontol. Series A, Biol Sci Med Sci 54: 203-211.

14. Lavallière $M$, Simoneau $M$, Tremblay $M$ et al (2012) Active training and driving-specific feedback improve older drivers' visual search prior to lane changes. BMC Geriatr 12: 5 .

15. Wilde GJS (1989) Accident countermeasures and behavioural compensation: The position of risk homeostasis theory. J Occupat Accid 10(4): 267-292.

16. Teasdale N, Lavallière M, Tremblay M, Simoneau M (2011) Older Drivers Fail to Come to a Complete Stop at Stop Sign-Controlled Intersections. Compte-rendu de la $21^{\mathrm{e}}$ Conférence canadienne multidisciplinaire sur la sécurité routière, Halifax, Nouvelle-Écosse, Canada

17. Salthouse TA (1996) The processing-speed theory of adult age differences in cognition. Psychol Rev 103(3): 403-428.

18. Makishita H, Matsunaga K (2008) Differences of drivers' reaction times according to age and mental workload. Accid Anal Prev 40(2): 567-575.

19. Rogé J, Pébayle T, Hannachi SE, Muzet A (2003) Effect of sleep deprivation and driving duration on the useful visual eld in younger and older subjects during simulator driving. Vision Res 43(13): $1465-1472$.
20. Rogé J, Pébayle T, Lambilliotte E et al. (2003) Inuence of age, speed and duration of monotonous driving task in traffic on the driver's useful visual eld. Vision Res 44(23): 2737-2744.

21. Sekuler R, Ball K (1986) Visual localization: age and practice. $J$ Opt Soc Am 3: 864-867.

22. Scialfa C, Kline D, Lyman B (1987) Age differences in target identication as a function of retinal location and noise level: examination of the useful eld of view. Psychol Aging 2: 14-19.

23. Ball K, Beard B, Roenker D et al. (1988) Age and visual search: expanding the useful eld of view. J Opt Soc Am 5(12): 2210-2219.

24. Crizzle AM, Myers AM (2013) Examination of naturalistic driving practices in drivers with Parkinson's disease compared to age and gender-matched controls. Acc Anal Prev 50: 724-731.

25. Marottoli RA, Mendes de Leon CF, Glass TA et al. (1997) Driving cessation and increased depressive symptoms: prospective evidence from the New Haven EPESE. Established Populations for Epidemiologic Studies of the Elderly. J Am Geriatr Soc 45: 202-206.

26. Wolinsky F, Johnson R, Stump T (1995) The risk of mortality among older adults over an eight-year period. The Gerontologist 35: $150-161$. 